\section{$\underset{\substack{\text { hommes } \\ \text { \& migrations }}}{ }$}

\section{Hommes \& migrations}

Revue française de référence sur les dynamiques

migratoires

$1305 \mid 2014$

L'exil chilien en France

\title{
Exil et littérature dans l'œuvre de Roberto Bolaño
}

Adélaïde de Chatellus

\section{(2) OpenEdition \\ Journals}

\section{Édition électronique}

URL : http://journals.openedition.org/hommesmigrations/2736

DOI : 10.4000/hommesmigrations.2736

ISSN : 2262-3353

\section{Éditeur}

Musée national de l'histoire de l'immigration

\section{Édition imprimée}

Date de publication : 1 janvier 2014

Pagination : 119-125

ISBN : 978-2919-040261

ISSN : $1142-852 X$

\section{Référence électronique}

Adélaïde de Chatellus, « Exil et littérature dans l'œuvre de Roberto Bolaño », Hommes \& migrations [En ligne], 1305 | 2014, mis en ligne le 01 janvier 2017, consulté le 19 avril 2019. URL : http://

journals.openedition.org/hommesmigrations/2736; DOI : 10.4000/hommesmigrations.2736 


\title{
EXIL ET LITTÉRATURE DANS L'EUURE DE ROBERTO BOLAÑO
}

par ADÉLAÏDE DE CHATELLUS, maître de conférences de littérature hispano-américaine, université de Paris-Sorbonne.

\author{
Roberto Bolaño occupe une place charnière dans I'histoire \\ de la littérature latino-américaine. Renonçant au réalisme \\ merveilleux ou magique qui a fait le succès de la génération \\ précédente, celle du Boom latino-américain, il développe \\ une œuvre marquée par l'abandon des utopies, de l'héroïsme, \\ de la morale. Chez lui, I'exil biographique croise \\ la volonté de renouveler la langue et l'esthétique littéraire \\ latino-américaines pour les ouvrir aux formes multiples \\ de la littérature universelle.
}

Roberto Bolaño est né en 1953 à Santiago du Chili. En 1968, à 15 ans, il part au Mexique pour suivre sa famille et y restera neuf ans. C'est là qu'il fait ses études et fonde l'infra-réalisme, un mouvement poétique mineur, avant-gardiste et libertaire. Poète, il publiera six recueils entre 1976 et 2007.

Les années 1970 sont pour lui synonyme d'engagement à gauche. Il devient trotskiste et repart au Chili pour soutenir le socialiste Salvador Allende. Mais le coup d'État du 11 septembre 1973 lui vaut d'être arrêté, soupçonné de terrorisme, et de passer huit jours à la prison de Concepción. Il quitte alors le Chili, et passe un moment au Salvador, en compagnie des guérilleros du Front Farabundo Martí de libération nationale, puis il revient à Mexico où il renoue avec sa vie de poète bohème.

\section{Une vie à Barcelone}

En 1977, à 24 ans, il arrive en Europe. D'abord brièvement en France, puis à Barcelone où il restera jusqu'à sa mort en 2003. Exilé, sans argent, il exerce au départ divers métiers pour vivre : il est alternativement groom, éboueur, plongeur et veilleur de nuit au camping Estrella del Mar de Castelldefels. En 1993, à 40 ans, lui est diagnostiqué un cancer du foie qui l'emportera dix ans plus tard. Vers la 
même date naissent ses deux enfants et - si l'œuvre d'un artiste ne saurait se réduire à sa biographie il est difficile de ne pas voir dans ces deux événements une explication possible à la publication tardive et accélérée de l'œuvre en prose. La production littéraire de Bolaño - éblouissante par sa qualité et sa quantité - coïncide, en effet, avec les dernières années de sa vie, comme si la conscience que le temps était compté avait Dans le cas de Bolaño, rendu urgentes l'écriture et l'exil fut donc à la fois subi (avec le coup d'état) et choisi, puisqu'à l'arrivée à Mexico avec ses parents il faut ajouter le choix de rester en Espagne à la chute de la dictature chilienne. la publication. Poète avant toute chose, ce n'est qu'à partir de la quarantaine qu'il publie de la prose et connaît la notoriété. C'est donc à Barcelone, et hors de l'Amérique latine, que l'essentiel de sa production voit le jour. Parue de son vivant ou posthume, elle comprend, entre autres, les romans La Littérature nazie en Amérique (1996), Étoile distante (1996), Les Détectives sauvages (1998), Amuleto (1999), Monsieur Pain (1999), Nocturne du Chili (2000), et le sublime 2666 (2004). Son œuvre inclut également des essais : Entre parenthèses (2004), Bolaño par luimême (2011), et des recueils de nouvelles : Appels téléphoniques (1997), Des putains meurtrières (2001) et Le Gaucho insupportable (2003) ${ }^{1}$.

\section{L'exil choisi et subi}

Dans le cas de Bolaño, l'exil fut donc à la fois subi (avec le coup d'État) et choisi, puisqu'à l'arrivée à Mexico avec ses parents il faut ajouter le choix de rester en Espagne à la chute de la dictature chilienne. Mort à 50 ans, il a passé trentecinq ans hors du Chili et seulement quinze dans son pays. Son parcours s'apparente à celui d'autres poètes chiliens de sa génération qui ont fui la dictature, comme Waldo Rojas qui s'établit à Paris, Raúl Barrientos aux États-Unis, où Naín Nómez à Toronto. Bolaño se situe également dans la tradition des écrivains latino-américains cosmopolites pour qui le passage par l'Europe reste déterminant. Ainsi, dans la génération qui le précède, celle dite du Boom latino-américain, le Cubain Carpentier, les Argentins Jorge Luis Borges, Julio Cortázar, Alejandra Pizarnik, les Mexicains Octavio Paz ou Carlos Fuentes et le Chilien Pablo Neruda sont passés par la France. Ou, dans la génération qui suit Bolaño, le Colombien Juan Gabriel Vázquez affirmait en 2011 avoir toujours eu la conviction qu'il devait aller en Europe pour devenir romancier et écrire sur son pays. À un journaliste qui l'interrogeait sur son identité, Bolaño répondit que sa seule patrie était la langue, et il ajouta: "Au Chili, personne n'a jamais dit que jétais chilien, on me disait toujours que jétais espagnol. En Espagne, il ne vient à l'idée d'aucun Espagnol que je suis espagnol. Au Mexique, il n'est jamais venu à l'idée de quiconque que j'étais mexicain. Par conséquent, jen suis arrivé à la conclusion que j’appartiens à un pays qui s'appelle Extrangilande, et dont les habitants sont les étrangers."." Qu'il soit choisi ou subi, l'exil fut un moteur de la création et prend dans l'œuvre de Bolaño la forme d'une distance esthétique multiple avec la littérature latino-américaine de sa jeunesse - celle des années 1960 à 1980 -, la littérature du Boom latino-américain ${ }^{3}$. Une distance esthétique qui fait de lui un écrivain charnière, un rénovateur de la littérature latino-américaine qui ouvre la voie à une génération nouvelle, celle des écrivains nés en Amérique latine à partir des années 1960, et qui voient en lui une idole.

\section{Un écrivain charnière : l'héritage du Boom latino-américain}

Les vingt-six ans passés hors de l'Amérique latine s'accompagnent d'une certaine distance avec la littérature latino-américaine de son temps. De cette dernière, Bolaño conserve l'ancrage géographique, avec des histoires situées majoritairement dans le 
monde hispanique. Mais - début d'émancipation - elles se passent dans plusieurs pays et rarement dans son pays natal. Ses nouvelles, dont les personnages ont un parcours souvent analogue au sien, se déroulent dans leur majorité à Barcelone ou au Mexique, parfois en France ou en Belgique, plus rarement dans les bas-fonds moscovites, quand un exilé russe vide son âme après sa bouteille dans un bar de Barcelone. Les nouvelles situées au Chili sont l'exception, ainsi "Enquêteurs", conversation de deux policiers de la dictature. Le roman Les Détectives sauvages est inspiré de la jeunesse bohème de Bolaño à Mexico, au point que Jorge Volpi parle de roman mexicain écrit à Barcelone par un Chilien. Le monumental 2666 se déroule à Ciudad Juárez, ville frontalière entre le Mexique et les États-Unis. Des textes qui portent trace de l'identité multiple de Bolaño et de son appartenance à plusieurs pays: "Ce n'est pas un hasard si Bolaño, un Chilien établi en Espagne, écrivait des nouvelles et des romans mexicains, chiliens, uruguayens, péruviens ou argentins avec le même naturel et la même conviction. Si les membres du Boom écrivaient des livres centrés sur leurs lieux d'origine respectifs dans le but d'évoquer l'insaisissable essence latino-américaine, Bolaño fit le contraire : écrire des livres qui jouaient à révéler le caractère fugitif de l'identité.".

\section{Une sensibilité de gauche}

Comme les auteurs la génération du Boom, Bolaño possède une sensibilité de gauche qui le pousse à dénoncer le cynisme de la dictature et à aborder la question du mal absolu. Ainsi, Étoile distante a pour protagoniste un personnage infâme, le poète Ruiz Tagle, qui, à l'arrivée de la dictature, révèle son vrai nom, Carlos Wieder, un serial killer qui prend des photos de ses victimes. De même, le personnage central de Nocturne du Chili est un prêtre de l'Opus Dei, critique littéraire et poète à l'agonie. Sur son lit de mort, il revient sur son passé, et le lec-

teur découvre progressivement ses liens avec la dictature. Les nouvelles de Bolaño se passent majoritairement dans les années 1970 dans un contexte de guerre froide. Les figures d'exilés chiliens au parcours chaotique y abondent, qui, comme l'auteur. ont parfois assisté à la torture dans les prisons de Pinochet. Des exilés chiliens ayant vécu au Mexique et menant à Barcelone une existence misérable où ils fréquentent leurs semblables. L'année 1973, celle de la chute d'Allende, revient de façon obsessionnelle (même dans les récits sans rapport avec le Chili), comme s'il s'agissait d'un traumatisme fondateur qui initierait un nouveau décompte du temps. Certains récits ont lieu pendant la Seconde Guerre mondiale ou dans la Russie soviétique. Comme chez les écrivains du Boom, la politique et l'Histoire sont donc présentes dans l'œuvre de Bolaño, mais l'écrivain introduit une tonalité nouvelle : l'abandon du messianisme et de la foi dans un

Comme les auteurs la génération du Boom Bolaño possède une sensibilité de gauche qui le pousse à dénoncer le cynisme de la dictature et à aborder la question du mal absolu. monde plus juste. Conscient d’appartenir à une génération sacrifiée, Bolaño a un rapport désabusé au politique, ne propose pas de solution, et ses textes sont parfois sans illusions sur les exilés: "Les exilés, des gens de gauche qui pensaient, du moins à partir de la taille et vers le bas, exactement pareil que les gens de droite qui en ce temps-là avaient fait main basse sur le Chili ."

\section{Une admiration pour la culture française}

Autre trait qui le rattache à la littérature de ses pères : une admiration sans limite pour la culture française. Poète, Bolaño vouait un culte à Rimbaud, aux poètes maudits et décadents, au surréalisme français et au cinéma de la Nouvelle Vague. Ce trait francophile est caractéristique des écrivains 
latino-américains du XXe siècle. Ainsi, tant le réalisme magique que le surréalisme latino-américain puisent à la source du surréalisme français, pour ne citer que deux exemples parmi bien d'autres. Une francophilie qui a disparu chez les écrivains latino-américains nés à partir de 1960, lesquels cherchent désormais leurs modèles dans la littérature anglo-saxonne.

\section{Un rénovateur de la littérature latino-américaine : le refus des idéologies}

S'il conserve certains traits de la littérature latinoaméricaine de sa génération, Bolaño ouvre la voie à des pistes nouvelles, avec des textes marqués par le refus des idéaux et du surnaturel et par un désir de renouvellement formel. Le refus de la morale est une variante du refus des frontières claires entre Bien et Mal. Les personnages de Bolaño mènent une vie de bohème, absorbent des drogues diverses - LSD, amphétamines et rohipnol ${ }^{7}$-, dérobent dans les librairies les livres qu'ils n'ont pas les moyens d'acheter ${ }^{8}$ ou donnent des conseils pour frauder dans les concours de nouvelles ${ }^{9}$. La recette est la suivante : envoyer le même texte à plusieurs concours car les membres ne sont jamais les mêmes d'un jury à l'autre. Et quand bien même le jury serait identique, il n'y a guère de risque puisque comme chacun sait - les jurys ne lisent pas. Il suffit donc d'envoyer le même texte en changeant de titre. C'est ainsi qu'une même nouvelle parviendra à un premier jury sous le titre "À l'aube", puis à un second sous celui de "Les Gauchos", pour un troisième elle sera intitulée "Dans l'autre pampa", et pour un dernier "Sans remords".

Le refus de la morale, ce sont aussi des relations amoureuses sans sentiment - et donc sans engagement -, qui oscillent entre le contact éphémère, la prostitution, la pornographie, le divorce, la solitude ou le meurtre passionnel.

\section{Refus de l'héroïsme}

Le rejet des utopies, c'est encore le refus de l'héroïsme au profit d'une fascination pour l'abîme. Les personnages de Bolaño sont des antihéros. Souvent exilés, pauvres comme Job, solitaires, sans profession véritable - seuls les acteurs pornographiques travaillent -, alternant les domiciles, les relations sociales, ce sont aussi des personnages qui refusent le système au point de lui préférer l'échec. L'humour de Bolaño aidant, abondent les figures d'écrivains ratés qui écrivent de mauvais textes dans des revues inconnues. Ainsi tel poète médiocre à qui le narrateur n'ose dire la vérité sur la qualité de ses écrits ${ }^{10}$. Des écrivains dont la médiocrité est généralement reconnaissable à leur admiration pour le poète espagnol Miguel Hernández et leur goût pour la souffrance ("Les mauvais poètes souffrent comme des animaux de laboratoire, surtout lors de leur longue jeunesse ${ }^{11}$ "). Des auteurs, enfin, qui siègent dans les prix littéraires de seconde zone, organisés par des caisses d'épargne, des municipalités, des chemins de fer, et dont les jurys sont éternellement les mêmes écrivains ratés ${ }^{12}$.

\section{Troubles mentaux et attrait pour les bas=fonds}

Les troubles mentaux appartiennent aussi au portrait de personnages blessés par la vie ou l'exil. Telle ex-amie du narrateur qui déprime et pense à des rats ${ }^{13}$; telle autre qui ne mange que de la puré $^{14}$. Tel exilé chilien qui se suicide après un passage par l'hôpital psychiatrique et une tentative de tuer sa femme ${ }^{15}$. Enfin, l'antihéroïsme, c'est l'attrait pour les bas-fonds, la pègre des narcotrafiquants, assassins, proxénètes et pornographes qui inspirent à Bolaño ses nouvelles les plus belles, dont certaines ont des accents semi-oniriques à 
Bolaño avait entre 20 et 40 ans. Comme beaucoup d'écrivains latino-américains d'aujourd'hui, Bolaño avait en horreur les clones du réalisme magique. Le réalisme magique est une esthétique dont García Márquez est souvent considéré comme le fondateur, avec le Cubain Carpentier qui lui donna le nom de "réalisme merveilleux". Ce ne sont pas eux, mais leurs imitateurs - et les bestsellers que ces derniers ont produits - que Bolaño exécrait : la Chilienne Isabel Allende, les Mexicaines Laura Esquivel ou Angeles Mastretta. Des auteurs de textes au succès planétaire en vertu desquels les mondes éditoriaux et universitaires étrangers ont trop souvent réduit la littérature latino-américaine à un univers exotique. Signe de ce ras-le bol, l'année 1996, date de parution de La Littérature nazie en Amérique et Étoile distante, est aussi celle de la publication de deux manifestes écrits par des écrivains de la génération suivante : d'une part, le manifeste du Crack signé par plusieurs écrivains mexicains nés à partir de 1960, désireux de revenir à une littérature complexe, exigeante avec le lecteur, à rebours de ces romans faciles. À cela s'ajoute la préface de l'anthologie de nouvelles MacOndo, publiée aussi en 1996 par les Chiliens

la Stanley Kubrick ${ }^{16}$. L'abandon des utopies, de l'héroïsme, de la morale ne laisse place quà deux formes de certitudes : l'humour et la passion pour la culture. Dans les textes de Bolaño abondent les références culturelles, en particulier poétiques et cinématographiques.

\section{Autre distance avec le Boom : le refus du surnaturel}

La distance avec la littérature du Boom latinoaméricain est aussi marquée par une esthétique réaliste qui refuse le surnaturel, pourtant marque de fabrique de la littérature du continent quand
Alberto Fuguet et Sergio Gómez, et qui présentait une nouvelle génération d'écrivains las d'être assimilés à une littérature exotique, rurale, ancrée en Amérique latine. Le refus du surnaturel, c'est aussi l'abandon du fantastique, autre marque de fabrique de la littérature latino-américaine du Boom, avec des auteurs comme Jorge Luis Borges, Adolfo Bioy Casares, Julio Cortázar, Silvina Ocampo, qui lui ont donné ses lettres de noblesse. Au fantastique, comme au réalisme magique, Bolaño oppose un réalisme sombre.

Textes qui abandonnent les idéologies et le surnaturel, les écrits de Bolaño donnent à l'exil la forme d'un éloignement thématique pluriel de la littérature latino-américaine de sa jeunesse. Mais l'exil, 
c'est aussi un désir de renouveau des formes qui mène à l'hybridation.

\section{L'hybridation formelle}

Le renouvellement formel, c'est aussi l'exploration du mélange des genres littéraires, avec des textes qui mêlent prose et poésie. Poète avant d'avoir été prosateur, Bolaño a une prose éminemment poétique, tandis que certains de ses poèmes sont narratifs. Ainsi la nouvelle "Le ver ${ }^{17 "}$ a-t-elle une variante rimée. L'hybridation touche enfin à la langue de Bolaño, un castillan imprégné de mexicanismes, de chilénismes, mais aussi de tournures propres à l'Espagne. On traite souvent des thèmes abordés par l'écrivain exilé, mais on oublie sa langue. Celle de Bolaño est un idiome qui porte trace de l'identité plurielle de l'auteur, un castillan pan-hispanique qui anticipe sur les expériences linguistiques de la génération née à partir de 1960.

\section{Un précurseur de la littérature latino-américaine d'aujourd'hui}

Enfin, par sa distance avec la littérature latino-américaine des années 1960 à 1980, Bolaño ouvrit la voie à la génération suivante, celle des écrivains nés à partir des années 1960. À la faveur de la mondialisation, ces derniers vivent par choix entre plusieurs pays et écrivent une littérature transnationale. Le Mexicain Jorge Volpi ; Andrés Neuman, né à Buenos Aires, qui a la double nationalité espagnole et argentine et réside à Grenade depuis l'âge de 15 ans ; Fernando Iwasaki Cauti, né au Pérou, de nationalité espagnole, avec un grand-père japonais et un autre italien ; Eduardo Halfón, né au Guatemala dans une famille fraîchement arrivée d'Israël, et qui écrit aux États-Unis des textes en langue espagnole ; Daniel Alarcón, de nationalité nord-américaine, né au Pérou, de langue maternelle espagnole, et qui, à l'inverse, écrit en anglais des œuvres entièrement situées dans son pays d'origine. Des écrivains aux esthétiques extrêmement variées, voire contradictoires, et qui n'ont souvent qu'un point commun, d'après Volpi : ils voient tous en Bolaño un gourou.

\section{Des œuvres situées ou non en Amérique latine}

Les œuvres de ces auteurs ne sont plus forcément situées en Amérique latine, la localisation devenant accessoire. Elles se situent parfois dans des pays sans rapport avec le monde hispanique. On pense à Amphytrion d'Ignacio Padilla et À la recherche de Klingsor de Jorge Volpi, deux romans sur l'Allemagne nazie. Les œuvres peuvent encore se situer dans un pays ou une ville sans nom, c'est-à-dire dans un cadre universel. Ainsi, les textes urbains du Vénézuélien Juan Carlos Méndez Guédez et du nord-américain Daniel Alarcón, lequel dit seetre servi de plusieurs villes du tiers monde pour élaborer son roman Lost City Radio. La variété des esthétiques aidant, il arrive parfois que les romans se situent en Amérique latine. C'est le cas quand ils ont l'Histoire pour toile de fond.

\section{Abandon du politique}

Dans ces textes récents, l'abandon du politique est manifeste. Bien souvent nés sous des dictatures, les auteurs ont assisté au retour de la démocratie qui n'a jamais été synonyme de justice, ni d'égalité, ni de prospérité, et, désabusés, ils ne croient plus aux utopies. Aucun d'entre eux n'a jamais été tenté par le militantisme. C'est là où ils poussent encore plus loin les tendances de l'œuvre et de la vie de Bolaño. Mais les injustices sont telles qu'il leur arrive de ne pas pouvoir se taire. Certaines œuvres abordent ainsi la violence d'État, mais elles en font une toile de fond. Elles se centrent sur la souffrance individuelle, sur les vies déstructurées par les guerres civiles ou sur la lutte contre le nar- 
cotrafic. Comme Bolaño, ces auteurs traitent du $\mathrm{Mal}$, de ses effets sur les citoyens, sans proposer d'utopie ni de remède.

\section{Expérimentation formelle}

Une caractéristique majeure de cette génération est l'expérimentation formelle, avec des textes hybrides qui non seulement mêlent les genres littéraires (prose, poésie, théâtre, essai) mais les ignorent, mêlant à la prose des formes d'expression non littéraire : nouvelle-SMS ou email, nouvelle-recette de cuisine, chanson, liste pour les courses, texte pictural ou musical, qui peuvent parfois inclure des images, etc. Ces auteurs poursuivent la voie tracée par Bolaño qui cherchait à renouveler la nouvelle et, à la suite de Borges ou de Rimbaud, abolissait les frontières entre prose et poésie.

\section{La langue : de l'espagnol de nulle part à l'anglais}

Certains auteurs nés à partir des années 1960 travaillent à abolir le dernier ancrage dans une nationalité : la langue. Certains, comme Andrés Neuman, élaborent un castillan de nulle part pour pousser le déracinement jusque dans la langue. D'autres, comme le Péruvien Daniel Alarcón ou le Dominicain Junot Diaz, arrivés enfants aux États-Unis, ont l'anglais comme langue d'expression, et non leur langue maternelle. Par son castillan trilingue, Bolaño préfigurait une des tendances de la littérature du continent qu'il est parfois difficile d'appeler latino-américaine. Au début des années 2000, on a parlé de littérature universelle en langue espagnole, mais les écrivains hispaniques anglophones des États-Unis ont brisé la dernière barrière identitaire : la littérature latino-américaine d'aujourd'hui ne s'écrit plus seulement en espagnol - elle s'écrit aussi en anglais -, ni en Amérique latine. Elle devient un défi à la dénomination au point qu’on pourrait parler de littérature post-nationale écrite par des écrivains nés en Amérique latine.

\section{Un écrivain universel}

Distance thématique et formelle avec les œuvres du Boom latino-américain, l'exil subi puis finalement choisi prend chez Bolaño la forme d'une ouverture vers une littérature universelle, et l'exploration de pistes transnationales qui ont tracé la voie de la génération suivante. L'exil de Bolaño est une contrainte qui, à l'instar de sa maladie, fut un moteur de la création, inséparable de son talent qui dépasse largement les frontières.

\section{Bibliographie}

Roberto Bolaño, Étoile distante, traduit de l'espagnol par Roberto Amutio, Paris, Christian Bourgois, 2002 [Estrella distante, Barcelona, Anagrama, 1996].

RobertoBolaño,Appelstéléphoniques, traduitdel'espagnol par Roberto Amutio, Paris, Christian Bourgois, 2004.

RobertoBolaño,NocturneduChili, traduitdel'espagnol par Roberto Amutio, Paris, Christian Bourgois, 2002 [Nocturno de Chile, Barcelona, Anagrama, 2000].

Roberto Bolaño, Des putains meurtrières, traduit de l'espagnol par Roberto Amutio, Paris, Christian Bourgois, 2003 [Putas asesinas, Barcelona, Anagrama, 2001].

Roberto Bolaño, "Sevilla me mata", in VV. AA., Palabra de América, Seix Barral, 2004, pp. 17-21.

Gustavo Faverón Patriau, Edmundo Paz Soldán, Bolaño salvaje, Barcelona, Candaya, 2008.

Jorge Volpi, "Bolaño, epidemia”, in Mentiras contagiosas, Madrid, Páginas de Espuma, 2008, pp. 235-251. Jorge Volpi, "Bolaño, perturbación", in El insomnio de Bolivar. Cuatro consideraciones intempestivas sobre América latina en el siglo XXI, Madrid, Debate, 2009, pp. 171-177. 